\title{
Subchondral bone plate sclerosis during late osteoarthritis is caused by loading-induced reduction in Sclerostin
}

\author{
Haoruo Jia, MD ${ }^{1,2, a}$, Xiaoyuan Ma, MD, PhD 31,1 , , Yulong Wei, MD¹, Wei Tong, MD, PhD ${ }^{1,4}$, \\ Robert J. Tower, $\mathrm{PhD}^{1}$, Abhishek Chandra, $\mathrm{PhD}^{1}$, Luqiang Wang, $\mathbf{M D}^{1}$, Zeyang Sun, $\mathbf{M S}^{1,5}$, \\ Zhaochun Yang, PhD $^{6}$, Farid Badar, BS ${ }^{7}$, Kairui Zhang, MD $^{8}$, Wei-ju Tseng, MS, MSE ${ }^{1}$, Ina \\ Kramer, PhD $^{9}$, Michaela Kneissel, PhD $^{9}$, Yang Xia, PhD $^{7}$, X. Sherry Liu, PhD ${ }^{1}$, James H-C. \\ Wang, $\mathrm{PhD}^{6}$, Lin Han, $\mathrm{PhD}^{10}$, Motomi Enomoto-Iwamoto, DDS, $\mathrm{PhD}^{11,12}$, and Ling Qin, \\ $\mathrm{PhD}^{1,{ }^{*}}$
}

${ }^{1}$ Department of Orthopaedic Surgery, School of Medicine, University of Pennsylvania, Philadelphia, Pennsylvania, 19104, USA 2Department of Orthopaedics, The First Affiliated Hospital of the Medical College, Shihezi University, ShiHeZi, Xinjiang, China ${ }^{3}$ Department of Orthopaedics, Shandong University Qilu Hospital, Jinan, Shandong, China ${ }^{4}$ Department of Orthopaedics, Union Hospital, Tongji Medical College, Huazhong University of Science and Technology, Wuhan, Hubei, China ${ }^{5}$ School of Engineering and Applied Science, University of Pennsylvania, Philadelphia, Pennsylvania, 19104, USA ${ }^{6}$ Department of Orthopaedic Surgery, University of Pittsburgh School of Medicine, Pennsylvania, 15213, USA 7Department of Physics and Center for Biomedical Research, Oakland University, Rochester, Michigan 48309, USA ${ }^{8}$ Department of Orthopaedics and Traumatology, Nanfang Hospital, Southern Medical University, Guangzhou, China ${ }^{9}$ Musculoskeletal Disease Area, Novartis Institutes for BioMedical Research, Basel, Switzerland ${ }^{10}$ School of Biomedical Engineering, Science and Health Systems, Drexel University, Philadelphia, Pennsylvania, 19104, USA ${ }^{11}$ Department of Surgery, The Children's Hospital of Philadelphia, Pennsylvania, 19104, USA ${ }^{12}$ Department of Orthopaedics, School of Medicine, University of Maryland, Baltimore, Maryland, 21201, USA

\section{Abstract}

Objective-To establish an unbiased 3D approach that quantifies subchondral bone plate (SBP) changes in mouse joints and to investigate the mechanism that mediates SBP sclerosis at a late stage of osteoarthritis (OA).

Design-A new microCT protocol was developed to characterize the entire SBP thickness in the distal femur of a mouse knee. Four mouse models with severe joint OA were generated: cartilagespecific Egfr knockout ( $C K O$ ) mice at 2 months after surgical destabilization of the medial meniscus (DMM), aged Egfr CKO mice, wild-type ( WT) mice at 10 months after DMM, and WT mice after DMM plus hemisectomy of the meniscus (DMMH) surgery. Additionally, SOST

*Corresponding author: Ling Qin, Department of Orthopaedic Surgery, University of Pennsylvania, G14A Stemmler Hall, $36^{\text {th }}$ Street and Hamilton Walk, Philadelphia, PA 19104, USA Tel: 215-8986697; Fax: 215-5732133; qinling@ pennmedicine.upenn.edu.

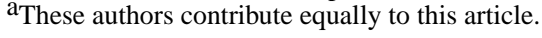

The authors have no conflict of interest. 
(Sclerostin) $\mathrm{KO}$ mice were subjected to DMMH surgery. Knee joints were examined by microCT, histology, and immunohistochemistry.

Results-3D microCT analysis of the mouse distal femur revealed a positive correlation between SBP thickness and the loading status in normal knees. In late OA models, SBP sclerosis was restricted to the areas under severely eroded articular cartilage. This was accompanied by elevated bone formation at the bone marrow side of the SBP and a drastic reduction of Sclerostin in osteocytes within the SBP. Unlike $W T$, SOST KO mice did not further increase SBP thickness in response to DMMH.

Conclusions-Since focal stress on SBP underlying the cartilage damage site increases at late OA, our work establishes mechanical loading-induced attenuation of Sclerostin and elevation of bone formation along the SBP surface as the major mechanism for subchondral bone phenotypes associated with late stage $\mathrm{OA}$ in mice.

\section{Introduction}

As the most common type of arthritis in the aging population, osteoarthritis (OA) is primarily characterized by progressive degeneration of knee articular cartilage. To date, most of the research seeking treatments for this disease has focused on prevention or repair of the degenerated cartilage. However, clinical observations and animal studies over the past several decades have accumulate sufficient evidence to support that OA is a whole joint disease involving changes not only in articular cartilage but also in its neighboring tissues, such as subchondral bone, ligaments, menisci, periarticular muscles, peripheral nerves, and synovium ${ }^{(1)}$. Indeed, the first radiographic criteria developed in 1950s for the diagnosis of OA include subchondral sclerosis as the hallmark of this disease ${ }^{(2)}$.

Anatomically, subchondral bone consists of two parts: a layer of corticalized subchondral bone plate (SBP) and the underlying subchondral trabecular bone (STB). It is important to distinguish these two subchondral bone components in OA research, not only due to their intrinsic architectural and mechanical differences, but also due to their different pathological changes during OA development ${ }^{(3)}$. Thickening of the SBP is considered an indisputable sign of late $\mathrm{OA}{ }^{(4)}$. However, the underlying mechanisms for such thickening are still largely unknown. In the STB, the bone remodeling rate is consistently increased during OA progression, leading to undermineralized bone tissue and reduced mechanical strength of trabecular bone ${ }^{(5-7)}$. Several mechanisms have been proposed to explain this phenomenon, such as cellular signaling for micro-damage repair, stimulation of vascular invasion, and bone-cartilage crosstalk via channels in the SBP ${ }^{(8)}$. Interestingly, recent studies showed that modifying osteoblastic gene expression or inhibiting osteoclastic bone resorption could prevent OA progression in mice ${ }^{(9)}$, suggesting that subchondral bone activity may control articular cartilage degeneration. Currently, it is still largely under debate whether subchondral bone sclerosis is the initiator or the consequence of cartilage damage.

Research on subchondral bone has been performed mostly on human OA samples and nonrodent animal models because of their large sizes. To elucidate the molecular mechanisms regulating the SBP and STB in OA, genetically modified mice are powerful tools. MicroCT is commonly used to characterize mouse subchondral bone changes. Generally, the proximal 
tibia is scanned at a relatively low resolution $(10-20 \mu \mathrm{m})$ and then contoured for 3D analysis of the STB. Due to its small size, the results might not be accurate. Measuring SBP thickness is particularly troublesome because it is usually performed on longitudinally sectioned 2D images. The results could be highly variable due to the inconsistency of sectioning angle and location. Furthermore, since it is based on 2D images, this approach does not capture changes in the entire SBP. Thus, there is an urgent need of designing an unbiased 3D approach to accurately quantify bone parameters of the SBP and STB in mouse joints.

In this study, we designed a protocol that is capable of measuring SBP thickness at any location within the mouse femoral condyle in a 3D manner, allowing comparisons among different anatomic sites (anterior vs posterior and medial vs lateral) in a 3D manner. Using this tool, we examined SBP changes in several types of mouse OA models: surgery-induced and aging models with genetically modified or non-modified mice. Biomechanical factors, such as those seen in obesity, trauma, and joint injury, play central roles in OA development and progression ${ }^{(10)}$. Sclerostin/Sost, an anti-osteogenic canonical Wnt inhibitor whose expression is largely restricted to osteocytes ${ }^{(11,12)}$, is an important mediator of mechanical loading-induced new bone formation ${ }^{(13)}$. Targeting this protein with a neutralizing antibody is currently being developed as a new therapy for osteoporosis ${ }^{(14)}$. Here, we explored the relationship among mechanical loading, Sclerostin level, and SBP thickness under normal and pathological conditions in these OA models. Our studies discovered a novel mechanism to explain how cartilage degeneration causes SBP sclerosis specifically underlying the cartilage damage site.

\section{Methods}

\section{Animals}

Egfr CKO (Col2-Cre Egfr $\left.{ }^{\text {Wa5/f }}\right)$ mice were generated as previously described ${ }^{(15)}$. Briefly, we first bred Col2a1-Cre with Egfr ${ }^{W 55 /+}$ to obtain Col2-Cre Egfr ${ }^{W 55 /+}$, which were then crossed with $E g f_{1}^{f / f}$ to generate Egfr CKO mice and their Wa5 (Egfr $\left.{ }^{\text {Wa5/f }}\right)$ and WT(Col2Cre $E g f r^{f / t}$ and $E g f r^{f /+}$ ) siblings. Wa5 is a dominant negative allele of $E g f f^{(16)}$ and is required for reducing EGFR activity in $C K O$ mice to a very low level ${ }^{(15)}$. We previously reported that Wa5 mice are morphologically and functionally closer to WTmice than $C K O$ mice, owning to the fact that the EGFR activity is only modestly decreased in Wa5 cells but drastically reduced in $C K O$ cells ${ }^{(15,17-19)}$. In this report, similar results were found, thereby all Wa5 mouse data were omitted from figures and results. Rosa-Tomato mice were purchased from Jackson Laboratory. To construct Egfr CKO Rosa-Tomato mice, we first obtained Egfr fff Rosa-Tomato homozygous mice and then used these mice to breed with Col2-Cre Egfr ${ }^{\text {Wa5/+ }}$ mice. Sclerostin (SOST) knockout (KO) mice were kindly provided by Novartis Pharma AG.

To induce OA, destabilization of the medial meniscus (DMM) or DMM plus hemisectomy of the meniscus (DMMH) surgery was performed on the right knees of 3-month-old or 2month-old male mice, respectively, as described previously ${ }^{(20,21)}$. Sham surgery was performed on the left knees. In accordance with the standards for animal housing, mice were group housed at $23-25^{\circ} \mathrm{C}$ with a $12 \mathrm{~h} \mathrm{light/dark} \mathrm{cycle} \mathrm{and} \mathrm{allowed} \mathrm{free} \mathrm{access} \mathrm{to} \mathrm{water} \mathrm{and}$ 
standard laboratory pellets. All animal work performed in this report was approved by the Institutional Animal Care and Use Committee (IACUC) at the University of Pennsylvania.

\section{Micro-computed tomography (microCT) analysis}

After euthanasia, mouse knee joints were harvested, fixed in $4 \%$ paraformaldehyde for 2 days, rinsed with running water, and stored in 1xPBS. A 3-mm region from the distal femur and the proximal tibia were then scanned at a 6- $\mu \mathrm{m}$ isotropic voxel size with a microCT 35 scanner (Scanco Medical AG, Brüttisellen, Switzerland). All images were smoothened by a Gaussian filter (sigma=1.2, support=2.0). For STB analysis, sagittal images were thresholded corresponding to $472.1 \mathrm{mg} \mathrm{HA} / \mathrm{cm}^{3}$ and contoured between the SBP and growth plate. Bone volume fraction (BV/TV), trabecular thickness (Tb.Th), trabecular separation (Tb.Sp), trabecular number (Tb.N), and structure model index (SMI) were calculated by 3D standard microstructural analysis ${ }^{(22)}$. For SBP analyses, sagittal images were contoured for the SBP followed by generating a 3D color map of thickness for the entire SBP. This map was converted to a grayscale thickness map, whose histogram was then used for quantification of the average SBP thickness at any defined area.

\section{Histology}

After microCT scans, knee joints were decalcified in 0.5 M EDTA (pH 7.4) for 4 weeks prior to paraffin embedding. A series of $6 \mu \mathrm{m}$-thick sagittal sections were cut across the entire medial and lateral compartments of the joint. The method to measure Mankin score was described previously ${ }^{(23)}$. Briefly, two sections within every consecutive six sections in the entire section set for each knee were stained with Safranin O/Fast green and scored by two blinded observers (HJ and XM). Each knee compartment received a single score representing the maximal score of its sections. Additional knee paraffin sections located at the middle of medial or lateral parts of joints were used for IHC. After antigen retrieval, slides were incubated with primary antibodies, such as rabbit anti-osteocalcin (Takara Clontech, m173) and goat anti-Sclerostin (R\&D Systems, AF1589), at $4^{\circ} \mathrm{C}$ overnight, followed by binding with biotinylated secondary antibodies and DAB color development. The images were captured using a Nikon Eclipse 90i microscope under the same conditions. For quantification of osteoblasts, we counted all osteocalcein ${ }^{+}$cells along the SBP surface at the bone marrow side underneath articular cartilage within the femoral-tibial contract region and normalized them against the length of SBP surface. For quantification of osteocytic Sclerostin staining intensity, the entire SBP region underneath articular cartilage was contoured and its staining intensity was quantified by the mean grey value after thresholding. Frozen sections were used for immunofluorescence staining. $90 \mu \mathrm{m}$-thickness frontal sections at the center of posterior region were incubated with endomucin antibody (Santa Cruz, sc-65495) at $4^{\circ} \mathrm{C}$ overnight, followed by Alexa Fluor ${ }^{\circledR} 488$-conjugated donkey anti-Rabbit IgG secondary antibody (Thermo Scientific, A-21206). The samples were scanned by confocal microscope LSM710 and images were processed and analyzed by Velocity software. The length of SBP surface at the bone marrow side that was aligned with blood vessels was measured and normalized against the total length of SBP surface. In all analyses, SBP region or surface that does not have overlying articular cartilage was excluded. 


\section{Statistical analysis}

Data are expressed as means \pm standard deviation (SD). Two-way ANOVA was performed when two independent variables (surgery: sham vs. DMM and genotype: $W T$ vs. $C K O$ ) were tested for comparisons. When there was a significant interaction effect between treatment and genotype, Mann-Whitney U-test with Bonferroni adjustment for multiple comparisons was performed to test difference between treatment groups within each genotypes as well as differences between genotypes within each group. In the scenario where a single independent variable was considered for comparisons, Mann-Whitney U-test was performed. Specifically, paired U-Tests were performed for comparisons between treatment (Sham vs. DMM) and between anatomic locations (anterior vs. posterior or medial vs. lateral) while unpaired U-tests were performed for comparison between mouse genotypes ( $W T$ vs. CKO or $K O$ ). Two-sided $\mathrm{p}$ value $<0.05$ were considered statistically significant.

\section{Results}

\section{A new microCT protocol reveals that SBP thickness is positively correlated with loading status in normal mice}

Examining 3D reconstructed microCT images of an adult mouse knee joint with a series of evenly divided frontal sections indicated that the distal femoral subchondral region is much larger and thicker than the proximal tibia subchondral region (Fig. 1A, B). On average, the height of the tibial STB was $180 \mu \mathrm{m}$ across the entire plateau. This limited height prevents precise segregation of the SBP and STB in most images, even when the bone is scanned at the highest resolution. In contrast, with an average of height around $600 \mu \mathrm{m}$, the femoral subchondral region is a better site for contouring STB for structural analysis despite sectioning planes.

Unlike humans, mouse knee joints are unable to fully extend. As shown in Fig. 1C, their bending angle is approximately between 30 and 150 degrees. This results in the posterior part, but not the anterior part, of distal femoral condyle being in direct contact with the tibial plateau, and therefore, is the area that undergoes load bearing and energy dissipation during joint motion. Interestingly, sagittal images implicated a thicker SBP at the posterior region relative to the anterior region (Fig. 1D). To quantify SBP thickness at any given area, we designed a new protocol to convert microCT images into a 3D color map, in which the color at any position linearly corresponds with its SBP thickness. Using this method, we can manually define the region of interest within the SBP and calculate the average thickness within this region (Fig. 1E). Quantification confirmed that the posterior SBP of femoral condyle is 2.2- and 1.6-fold thicker than the anterior SBP region at the medial and lateral sites, respectively (Fig. 1F). This result provides the first line of evidence that SBP thickness is positively correlated with loading status. Since the SBP is very close to the growth plate in the tibia, it is difficult to apply this approach to the tibia. Therefore, we focused on the distal femur for STB and SBP analyses in our studies.

\section{Egfr CKO mice develop SBP sclerosis at the medial posterior site after DMM}

We previously reported that EGFR is critical for maintaining superficial chondrocyte number, promoting cartilage surface lubrication, and retaining cartilage mechanical 
functions ${ }^{(17)}$. Strikingly, Egfr CKO (Col2-Cre Egfr $\left.{ }^{W a 5 / f}\right)$ mice developed severe OA phenotype at 2-3 months after DMM with a complete depletion of the articular cartilage layer at the medial posterior site (Fig. S1A). Note that the cartilage remained intact at the medial anterior site in both $W T$ and $C K O$ mice after DMM (Fig. S1B). MicroCT analysis of the femoral STB detected a modest but significant decrease in BV/TV, accompanied by reduced trabecular thickness in $C K O$, but not in $W T$ mice, after DMM (Fig. S2). Interestingly, OA progression induced a drastic increase in SMI of STB in $C K O$ mice, indicating a deterioration of trabecular structure at late OA.

The 2D frontal microCT images (Fig. 2A) implied that the SBP at the medial site of $C K O$ femur is substantially thickened after DMM. By applying our new protocol, we calculated SBP thickness at 4 locations within the femoral condyle (medial posterior, lateral posterior, medial anterior, and lateral anterior). Interestingly, we observed a significant increase in SBP thickness only at the medial posterior site (31\%) in $C K O$ mice after DMM, where cartilage degradation was the most severe (Fig. S1). This thickening is absent at other sites in $C K O$ mice and at all four sites in $W T$ mice (Fig. 2B, C), implicating that this localized SBP response is likely due to the depletion of overlying articular cartilage.

\section{SBP thickening in Egfr CKO mice after DMM coincides with increased bone formation at the bone marrow side of the SBP and reduced Sclerostin level in the SBP}

To elucidate the mechanism of SBP thickening, we first investigated where new bone is formed. Osteocalcin staining revealed that the number of osteoblasts lining the SBP at the bone marrow side increases 9.7- and 8.6-fold at DMM medial site of $C K O$ femur compared to those at sham medial and DMM lateral sites, respectively (Fig. 3A, B). This enhanced bone formation was accompanied by an increased length of blood vessels lining the SBP surface (Fig. 3C, D), likely providing more oxygen and nutrients needed for active bone formation.

Wnts are potent signals for osteoblastic bone formation. Sclerostin is a Wnt pathway antagonist that is almost exclusively secreted by osteocytes ${ }^{(12)}$. Interestingly, its expression is mainly regulated by mechanical stimulation on bone ${ }^{(13)}$. It is commonly accepted that in normal joints, the meniscus plays a key role in reducing the contact stresses on articular cartilage ${ }^{(24)}$ and that a loss of cartilage at late OA results in greatly amplified focal stresses on the underlying subchondral bone ${ }^{(25)}$. Therefore, we hypothesized that Sclerostin expression is specifically down-regulated in the SBP under the damaged cartilage after DMM due to abnormal overloading. Like those in the cortical bone, osteocytes in the SBP express high amounts of Sclerostin within cell bodies and throughout dendritic processes (Fig. 3E), indicating a possible role for Sclerostin in maintaining the SBP. In WT mice, DMM did not alter Sclerostin levels at either medial or lateral sites (Fig. 3E top, F).

Strikingly, in $C K O$ mice, Sclerostin amounts were markedly attenuated within the SBP at the DMM medial site, but not at the DMM lateral site (Fig. 3E bottom, F). These results suggest that a loss of Sclerostin in the SBP underneath the damaged cartilage at late OA stage may play a role in new bone formation at the bone marrow side of the SBP. 


\section{Aged Egfr CKO mice share the same mechanism of SBP thickening as Egfr CKO DMM model}

Egfr CKO mice developed spontaneous OA with no SBP phenotype at 6 months of age ${ }^{(17)}$. At 12 months of age, while OA phenotypes in articular cartilage continued (Fig. 4A, B), their STB undergoes significant bone loss accompanied with deterioration in structure (Fig. S3). Note that unlike surgery-induced OA, age-related OA exhibits cartilage damage at both medial and lateral regions. This caused a similar level of SBP sclerosis at the posterior site across the entire distal femur in Egfr CKO mice (Fig. 4C, D). WT knees remained healthy at this age. We found that osteoblast number is elevated by 4.3- and 5.4-fold at medial and lateral sites, respectively, in $C K O$ compared to $W T$ (Fig. 4E, F). Dynamic histomorphometry revealed that newly formed bone surfaces are only observed at the bone marrow side of the SBP but not at the cartilage side (Fig. S4A) and that bone formation rate (BFR) was elevated by 3.5-fold at both medial and lateral sites of $C K O$ femurs (Fig. S4B, C). Furthermore, Sclerostin levels in SBP osteocytes were significantly decreased at medial and lateral sites in $C K O$ compared to $W T$ (Fig. 4G, H).

\section{SBP sclerosis in other late OA models}

To confirm that our conclusion is not restricted to Egfr $C K O$ mice, we tested two late OA models in $W T$ mice. At 10 months after DMM surgery, $W T$ mice exhibited severe OA phenotypes including a complete depletion of articular cartilage (Fig. 5A, B) and a trend of bone loss in the STB (Fig. S5). In line with our Egfr CKODMM model, these mice displayed SBP thickening only at the medial posterior site, but not at the other 3 sites in the femoral condyle (Fig. 5C, D). Staining confirmed a reduction in osteocytic Sclerostin levels within thickened SBP after DMM (Fig. 5E, F).

Compared to DMM, which produces only moderate OA, DMMH causes more severe OA ${ }^{(20)}$. At 14 weeks post-surgery, WT joints had already developed late OA phenotypes with severe cartilage degeneration at the medial posterior site (Fig. 6A, B). No significant signs of cartilage degeneration were observed in sham joints. Consequently, they exhibited SBP thickening only at the medial posterior site but not at the lateral posterior site (Fig. 6C, D). Again, we observed a significant decrease in Sclerostin only at the medial site of DMMH knees but not at the lateral site (Fig. 6F). To determine whether Sclerostin inhibition mediates SBP sclerosis, we performed DMMH on SOST KO mice. Consistent with a previous report ${ }^{(26)}$, in sham joints, SOST KO mice had much more subchondral bone than $W T$ mice. Strikingly, while their DMMH medial posterior site developed a similar level of cartilage degeneration as $W T$ mice (Fig. 6A, B), the SBP thickness was the same as sham joints (Fig. 6C, E). Taken together, our data from these OA models support that SBP sclerosis is likely the result of reduced osteocytic Sclerostin in the SBP during OA progression.

\section{Discussion}

Abnormal mechanical loading plays a pivotal role in causing OA ${ }^{(27)}$. To date, major risk factors for knee OA are identified as older age, female, obesity, knee injury, and occupational overuse ${ }^{(28,29)}$. While the first two indicate the vulnerability of the knee to 
injury, the latter three represent either chronic, excessive loading or focally increased stress on the knee. Most previous studies focus on the mechanobiology of chondrocytes and cartilage tissue to understand the role of overloading in the initiation and progression of OA ${ }^{(30)}$. In OA patients, it has been long observed that subchondral bone is abnormal with increased bone resorption (31). Taking advantage of four genetically modified and $W T$ mouse models that develop severe OA, we demonstrate that abnormal over-loading, caused by depletion of the overlying cartilage, attenuates osteocytic Sclerostin within the SBP and subsequently increases bone formation at the bone marrow side of the SBP presumably via activating Wnt signaling. Thus, our work elucidates the intimate relationship among mechanical loading, osteocytic Sclerostin, and SBP sclerosis at late stage OA. This conclusion is further supported by a recent patient study showing that Sclerosin levels within the SBP is negatively correlated with OA severity ${ }^{(32)}$.

A prerequisite for drawing our conclusion is our new microCT protocol that calculates SBP thickness in 3D across the entire femoral epiphyseal area. This method eliminates bias arising from sectioning angle and location. Moreover, this approach allows us to compare the same region among different bones and different anatomical sites within the same bone, thus decreasing sample variations. Due to the small size of mouse joints, we have demonstrated that the distal femur is a better site than the proximal tibia for subchondral bone analysis under normal condition. After DMM, SBP thickening in the tibia often results in a fusion of the SBP with the growth plate, making it impossible to analyze bone structure.

Several lines of evidence support our conclusion. First, analyzing distal femoral epiphyseal bone revealed that the load-bearing posterior site is thicker than the non-load-bearing anterior site of the same bone, regardless of medial or lateral location, indicating that SBP thickness is positively correlated with the loading applied to it. Second, we have tested four distinct mouse models with either severe OA or long-term chronic OA symptoms. In all models, we observed a substantial decrease in Sclerostin levels expressed by osteocytes within the thickened SBP underlying damaged articular cartilage. This phenomenon is location specific. In surgery models (Egfr CKO mice at 2 months after DMM, WT mice at 10 months after DMM and at 14 weeks after DMMH), the SBP was only expanded, and Sclerostin amount was only attenuated at the medial posterior site, where cartilage damage was concentrated. The SBP responses were not detected at the lateral posterior site, where cartilage damage was modest at most. In contrast, since OA-induced cartilage degeneration was developed chronically at both medial and lateral sites in aged Egfr CKO model, alterations in the SBP were correspondently observed at both lateral and medial posterior sites. Third, despite changes at the posterior region, the anterior SBP thickness, regardless of medial or posterior sites, remained the same in our OA models. Lastly, data from SOST KO mice after DMMH surgery support that Sclerostin mediates SBP thickening underneath the eroded cartilage at late OA stage. Repetitive mechanical loading on diaphyseal cortical bone reduces Sclerostin expression to achieve anabolic bone formation ${ }^{(33)}$. Indeed, the SBP is structurally similar to cortical bone and has similarly higher Sclerostin levels than trabecular bone (data not shown). It is very likely that suppressed Sclerostin expression observed in the SBP underneath the damaged cartilage is owing to the increased focal stress on the SBP. Therefore, our data provide another piece of evidence supporting the role of Sclerostin in the 
bone responses toward mechanical loading. Taken together, we demonstrate a critical role of mechanics in shaping the SBP after OA and elucidate the underlying molecular mechanism.

Interestingly, in addition to SBP thickening, Egfr CKO OA models became modestly osteopenic in the STB. Our previous report has shown that metaphyseal trabecular bone and serum bone markers are not affected in Egfr CKO mice (17), indicating that overall bone remodeling is not altered due to the genetic modification of Egfr in cartilage. This result is consistent with a previous statement that OA-induced bone damage is restricted to the subchondral bone region ${ }^{(34)}$. We believe that defects in articular cartilage are sufficient to induce subchondral bone abnormalities under enhanced mechanical loads.

Whether subchondral bone sclerosis is a driving force for cartilage degeneration or a consequence of cartilage degeneration has been disputed for many years. Since our DMM surgery develops modest OA in $W T$ mice, we only detected significant changes in the SBP and STB at 10 months after surgery. At 2 months after surgery, while DMM already generates changes in the articular cartilage, such as partial loss of proteoglycan and surface fibrillation, it has no detectable effect on subchondral bone structure in these mice. All subchondral bone responses in our models require either a substantial period of time after $\mathrm{OA}$ initiation or a $C K O$ mouse model that is predisposed for OA development. Moreover, our previous report reveals that cartilage surface modulus at future OA site is substantially reduced at 1 week after DMM surgery, owing to increased catabolic activities ${ }^{(35)}$. This time point is much earlier than any detectable cartilage morphological changes, let alone subchondral bone alterations. Hence, our data strongly support that subchondral bone sclerosis is secondary to cartilage degeneration.

We are also interested in exploring whether changes in subchondral bone exaggerate overlying cartilage degeneration. SOST KO mice have substantially more trabecular and cortical bone than $W T^{(36)}$. In 1-year-old SOST KO mice, the STB robustly expands, resulting in a solid bone between articular cartilage and the growth plate (data not shown). Consistent with a previous report ${ }^{(37)}$, we did not detect any cartilage degeneration in these mice from 3-month-old up to 14-month-old (data not shown). Four weeks of Sclerostinantibody (Scl-Ab) injections in WT mice also does not alter articular cartilage while it drastically increases BV/TV in the STB and thickens the SBP (data not shown). In contrast to a previous report showing accelerated OA in SOST KO mice after DMM ${ }^{(26)}$, we found that DMMH surgery causes similarly severe OA symptoms in WT and SOST KO mice. Furthermore, intermittent injections of parathyroid hormone (PTH) 1-34 peptide, a potent anabolic agent for stimulating bone formation and bone turnover, has been shown to either attenuate OA progression ${ }^{(38)}$ or have no effect in surgery-induced OA models ${ }^{\left({ }^{39}\right)}$. In our hands, PTH injections quickly enlarged the SBP within 2-3 weeks but did not accelerate OA progression after DMM (data not shown). More importantly, no clinical report has shown a positive correlation between PTH treatment and OA incidents in osteoporosis patients. From a mechanical point of view, thickening of the SBP from the bone marrow side, as we have demonstrated as the major site for new bone formation during OA, is unlikely to affect the load distribution at the surface of overlying cartilage. We believe that SBP thickening alone is not sufficient to affect cartilage degeneration, and therefore, cannot be an initiator of 
cartilage and joint damage in OA development. Whether bone alterations in the STB caused by enhanced bone turnover contribute to OA progress is still not clear from our studies.

Our studies have several limitations. First, our sham surgery was performed in the contralateral joint rather than in a separate group of mice. Though we did not detect any obvious OA changes in sham joints, animals may still have altered loading patterns and gaits. Second, DMMH was performed on skeletal immature mice. We chose this age (8 weeks) because the SBP of SOST KO is continuously thickened throughout the life, causing difficulties in accurately measuring its thickness in older mice. Third, it is possible that the BFR at the bone marrow side of the SBP of SOST KO has already reached the maximum, and so cannot be further enhanced by the increased loading after cartilage depletion.

However, PTH injections have been shown to increase the high basal levels of periosteal and endosteal BFR in the femoral midshaft of SOST KO mice (40), suggesting that the BFR of SOST KOSBP can be further elevated. Lastly, all our data come from mouse models. While mouse has many advantages in cartilage research, particularly in discovering novel cellular and molecular mechanisms, studies on large animal models are required before translating our finding in designing future clinical applications. In summary, our work establishes mechanical loading-induced attenuation of Sclerostin and elevation of bone formation along the SBP as the major mechanism for subchondral bone sclerosis associated with late OA. Thus, correcting the abnormal loading might be an effective approach for attenuating subchondral bone damage in OA patients.

\section{Supplementary Material}

Refer to Web version on PubMed Central for supplementary material.

\section{Acknowledgments}

This study was supported by ASBMR Research Career Enhancement Award (to LQ), NIH grants AR060991, DK095803 (to LQ), AR062908 (to ME-I), AR069047 (to YX), AR065949 (to JHW), AR066824 (to LH), and the Penn Center for Musculoskeletal Disorders Histology Core (P30-AR06919).

\section{References}

1. Lane NE, Brandt K, Hawker G, et al. OARSI-FDA initiative: defining the disease state of osteoarthritis. Osteoarthritis Cartilage. 2011; 19(5):478-82. [PubMed: 21396464]

2. Kellgren JH, Lawrence JS. Radiological assessment of osteo-arthrosis. Ann Rheum Dis. 1957; 16(4):494-502. [PubMed: 13498604]

3. Dedrick DK, Goldstein SA, Brandt KD, O’Connor BL, Goulet RW, Albrecht M. A longitudinal study of subchondral plate and trabecular bone in cruciate-deficient dogs with osteoarthritis followed up for 54 months. Arthritis Rheum. 1993; 36(10):1460-7. [PubMed: 8216405]

4. Goldring SR, Goldring MB. Changes in the osteochondral unit during osteoarthritis: structure, function and cartilage-bone crosstalk. Nat Rev Rheumatol. 2016; 12(11):632-44. [PubMed: 27652499]

5. Bailey AJ, Mansell JP, Sims TJ, Banse X. Biochemical and mechanical properties of subchondral bone in osteoarthritis. Biorheology. 2004; 41(3-4):349-58. [PubMed: 15299267]

6. Bettica P, Cline G, Hart DJ, Meyer J, Spector TD. Evidence for increased bone resorption in patients with progressive knee osteoarthritis: longitudinal results from the Chingford study. Arthritis Rheum. 2002; 46(12):3178-84. [PubMed: 12483721] 
7. Funck-Brentano T, Cohen-Solal M. Crosstalk between cartilage and bone: when bone cytokines matter. Cytokine Growth Factor Rev. 2011; 22(2):91-7. [PubMed: 21596615]

8. Burr DB, Gallant MA. Bone remodelling in osteoarthritis. Nat Rev Rheumatol. 2012; 8(11):665-73. [PubMed: 22868925]

9. Findlay DM, Kuliwaba JS. Bone-cartilage crosstalk: a conversation for understanding osteoarthritis. Bone Res. 2016; 4:16028. [PubMed: 27672480]

10. Arden N, Nevitt MC. Osteoarthritis: epidemiology. Best Pract Res Clin Rheumatol. 2006; 20(1):325. [PubMed: 16483904]

11. Poole KE, van Bezooijen RL, Loveridge N, et al. Sclerostin is a delayed secreted product of osteocytes that inhibits bone formation. Faseb J. 2005; 19(13):1842-4. [PubMed: 16123173]

12. van Bezooijen RL, Roelen BA, Visser A, et al. Sclerostin is an osteocyte-expressed negative regulator of bone formation, but not a classical BMP antagonist. J Exp Med. 2004; 199(6):805-14 [PubMed: 15024046]

13. Galea GL, Lanyon LE, Price JS. Sclerostin's role in bone's adaptive response to mechanical loading. Bone. 2017; 96:38-44. [PubMed: 27742499]

14. McClung MR, Grauer A, Boonen S, et al. Romosozumab in postmenopausal women with low bone mineral density. N Engl J Med. 2014; 370(5):412-20. [PubMed: 24382002]

15. Zhang X, Zhu J, Li Y, et al. Epidermal growth factor receptor (EGFR) signaling regulates epiphyseal cartilage development through beta-catenin-dependent and -independent pathways. J Biol Chem. 2013; 288(45):32229-40. [PubMed: 24047892]

16. Lee D, Cross SH, Strunk KE, et al. Wa5 is a novel ENU-induced antimorphic allele of the epidermal growth factor receptor. Mamm Genome. 2004; 15(7):525-36. [PubMed: 15366372]

17. Jia H, Ma X, Tong W, et al. EGFR signaling is critical for maintaining the superficial layer of articular cartilage and preventing osteoarthritis initiation. Proc Natl Acad Sci U S A. 2016; 113(50):14360-5. [PubMed: 27911782]

18. Zhang X, Siclari VA, Lan S, et al. The critical role of the epidermal growth factor receptor in endochondral ossification. Journal of bone and mineral research : the official journal of the American Society for Bone and Mineral Research. 2011; 26(11):2622-33.

19. Zhang X, Tamasi J, Lu X, et al. Epidermal growth factor receptor plays an anabolic role in bone metabolism in vivo. Journal of bone and mineral research : the official journal of the American Society for Bone and Mineral Research. 2011; 26(5):1022-34.

20. Kamekura S, Hoshi K, Shimoaka T, et al. Osteoarthritis development in novel experimental mouse models induced by knee joint instability. Osteoarthritis Cartilage. 2005; 13(7):632-41. [PubMed: 15896985]

21. Zhang X, Zhu J, Liu F, et al. Reduced EGFR signaling enhances cartilage destruction in a mouse osteoarthritis model. Bone Research. 2014; 2:14015. [PubMed: 26120493]

22. Bouxsein ML, Boyd SK, Christiansen BA, Guldberg RE, Jepsen KJ, Muller R. Guidelines for assessment of bone microstructure in rodents using micro-computed tomography. J Bone Miner Res. 2010; 25(7):1468-86. [PubMed: 20533309]

23. Aigner T, Cook JL, Gerwin N, et al. Histopathology atlas of animal model systems - overview of guiding principles. Osteoarthritis Cartilage. 2010; 18(Suppl 3):S2-6. [PubMed: 20864020]

24. Walker PS, Erkman MJ. The role of the menisci in force transmission across the knee. Clin Orthop Relat Res. 1975; 109:184-92.

25. Eberhardt AW, Keer LM, Lewis JL, Vithoontien V. An analytical model of joint contact. J Biomech Eng. 1990; 112(4):407-13. [PubMed: 2273867]

26. Bouaziz W, Funck-Brentano T, Lin H, et al. Loss of sclerostin promotes osteoarthritis in mice via beta-catenin-dependent and -independent Wnt pathways. Arthritis Res Ther. 2015; 17(1):24. [PubMed: 25656376]

27. Felson DT. Osteoarthritis as a disease of mechanics. Osteoarthritis Cartilage. 2013; 21(1):10-5. [PubMed: 23041436]

28. Blagojevic M, Jinks C, Jeffery A, Jordan KP. Risk factors for onset of osteoarthritis of the knee in older adults: a systematic review and meta-analysis. Osteoarthritis Cartilage. 2010; 18(1):24-33. [PubMed: 19751691] 
29. Felson DT. Obesity and vocational and avocational overload of the joint as risk factors for osteoarthritis. J Rheumatol Suppl. 2004; 70:2-5. [PubMed: 15132347]

30. Sanchez-Adams J, Leddy HA, McNulty AL, O'Conor CJ, Guilak F. The mechanobiology of articular cartilage: bearing the burden of osteoarthritis. Curr Rheumatol Rep. 2014; 16(10):451. [PubMed: 25182679]

31. Hunter DJ, Hart D, Snieder H, Bettica P, Swaminathan R, Spector TD. Evidence of altered bone turnover, vitamin $\mathrm{D}$ and calcium regulation with knee osteoarthritis in female twins. Rheumatology (Oxford). 2003; 42(11):1311-6. [PubMed: 12867590]

32. Wu L, Guo H, Sun K, Zhao X, Ma T, Jin Q. Sclerostin expression in the subchondral bone of patients with knee osteoarthritis. Int J Mol Med. 2016; 38(5):1395-402. [PubMed: 27665782]

33. Robling AG, Niziolek PJ, Baldridge LA, et al. Mechanical stimulation of bone in vivo reduces osteocyte expression of Sost/sclerostin. J Biol Chem. 2008; 283(9):5866-75. [PubMed: 18089564]

34. Brown TD, Radin EL, Martin RB, Burr DB. Finite element studies of some juxtarticular stress changes due to localized subchondral stiffening. J Biomech. 1984; 17(1):11-24. [PubMed: 6715384]

35. Doyran B, Tong W, Li Q, et al. Nanoindentation modulus of murine cartilage: a sensitive indicator of the initiation and progression of post-traumatic osteoarthritis. Osteoarthritis Cartilage. 2017; 25(1):108-17. [PubMed: 27568574]

36. Chandra A, Lin T, Young T, et al. Suppression of Sclerostin Alleviates Radiation-Induced Bone Loss by Protecting Bone-Forming Cells and Their Progenitors Through Distinct Mechanisms. J Bone Miner Res. 2017; 32(2):360-72. [PubMed: 27635523]

37. Roudier M, Li X, Niu QT, et al. Sclerostin is expressed in articular cartilage but loss or inhibition does not affect cartilage remodeling during aging or following mechanical injury. Arthritis Rheum. 2013; 65(3):721-31. [PubMed: 23233270]

38. Sampson ER, Hilton MJ, Tian Y, et al. Teriparatide as a chondroregenerative therapy for injuryinduced osteoarthritis. Sci Transl Med. 2011; 3(101):101ra93.

39. Bagi CM, Berryman E, Zakur DE, Wilkie D, Andresen CJ. Effect of antiresorptive and anabolic bone therapy on development of osteoarthritis in a posttraumatic rat model of OA. Arthritis Res Ther. 2015; 17:315. [PubMed: 26542671]

40. Robling AG, Kedlaya R, Ellis SN, et al. Anabolic and catabolic regimens of human parathyroid hormone 1-34 elicit bone- and envelope-specific attenuation of skeletal effects in Sost-deficient mice. Endocrinology. 2011; 152(8):2963-75. [PubMed: 21652726] 
A $3 D$

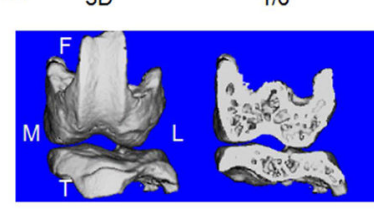

$2 / 6$

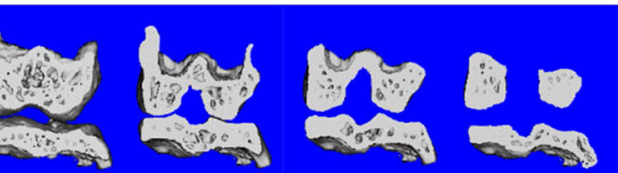

B

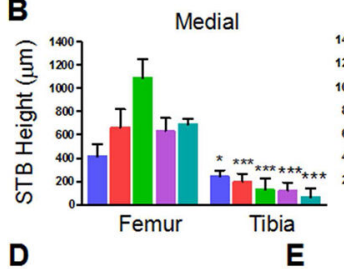

D

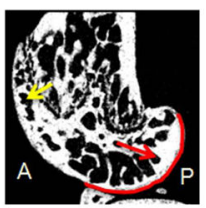

E

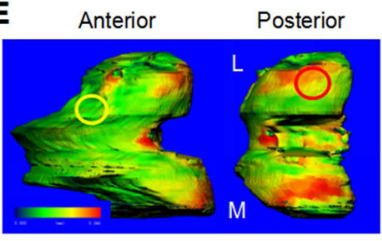

C $30^{\circ} \quad 150^{\circ}$
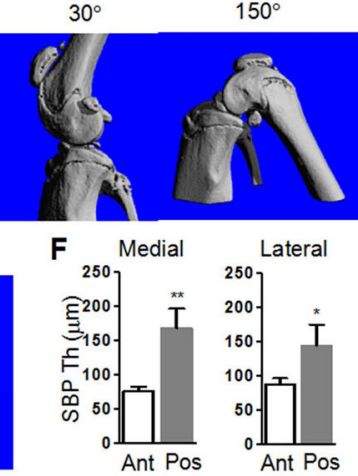

Figure 1.

Characterization of mouse subchondral bone using a new microCT protocol.

(A). A 3D-reconstructed 4-month-old WT mouse knee was completely sectioned along the frontal plane. Images corresponding to $1 / 6,2 / 6,3 / 6,4 / 6$, and 5/6 positions from the most anterior part (0) to the most posterior part (1) were shown. M: medial; L: lateral; F: femur; T: tibia.

(B). Heights of femoral and tibial STB at the middle of medial and lateral regions of $1 / 6$ to $5 / 6$ positions were quantified. $\mathrm{n}=6$ mice. $*: \mathrm{p}<0.05$, ***: $\mathrm{p}<0.001$ vs the corresponding level of femur.

(C). The bending angle between tibia and femur in a mouse knee is between $30^{\circ}$ and $150^{\circ}$.

(D). A sagittal image of a femur epiphyseal region reveals that only the posterior site of the SBP (red lines) is the load-transducing site and that SBP is thicker at the posterior (A) site (red arrow) than the anterior (A) site (yellow arrow).

(E). 3D color maps of SBP thickness show the view from anterior and posterior angles. Yellow and red circles indicate the regions for thickness calculation at medial anterior and medial posterior sites, respectively. Color ranges from 0 (blue) to $246 \mu \mathrm{m}$ (red).

(F) Quantification of SBP thickness at four distinct sites in femoral condyle. $\mathrm{n}=5$ mice. *: $\mathrm{p}<0.05, * *: \mathrm{p}<0.01$ vs Ant. 


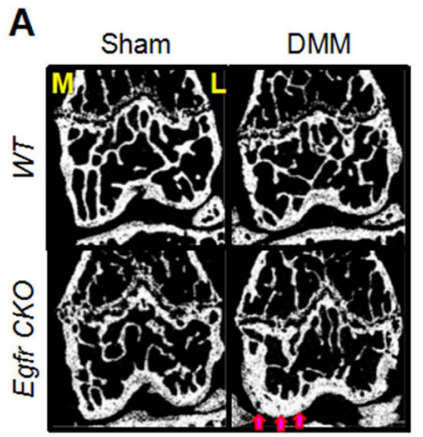

B
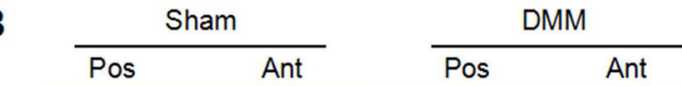

C
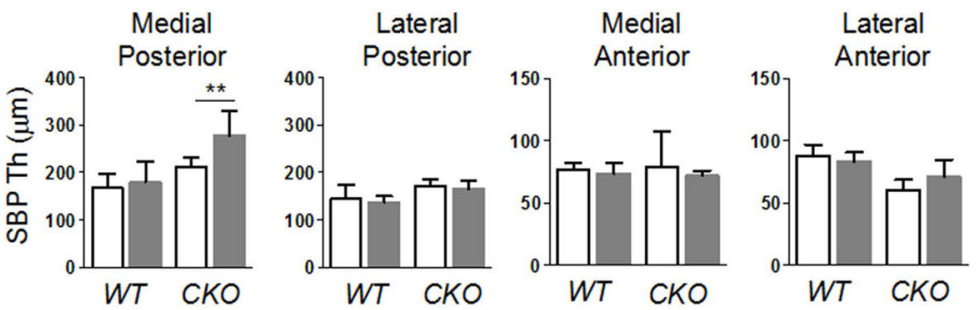

Figure 2.

Egfr $C K O$ mice develop local SBP sclerosis underneath the severely damaged cartilage area after OA surgery.

(A). Representative frontal microCT images of $W T$ and $C K O$ femoral epiphyseal region at 2 months post-surgery. M: medial; L: lateral. Red arrows point to thickened SBP at the medial site of $C K O$ joint after DMM.

(B). Representative 3D color maps of SBP thickness in $W T$ and $C K O$ femurs with or without DMM. Color ranges from 0 (blue) to $430 \mu \mathrm{m}$ (red). Pos: posterior; Ant: anterior. (C). SBP thickness at four distinct sites in the femur was quantified. $n=5$ mice/genotype. 2 way ANOVA shows $\mathrm{p}<0.05$ for the interaction between surgery and genotype at medial posterior site. $* *: \mathrm{p}<0.01$. 

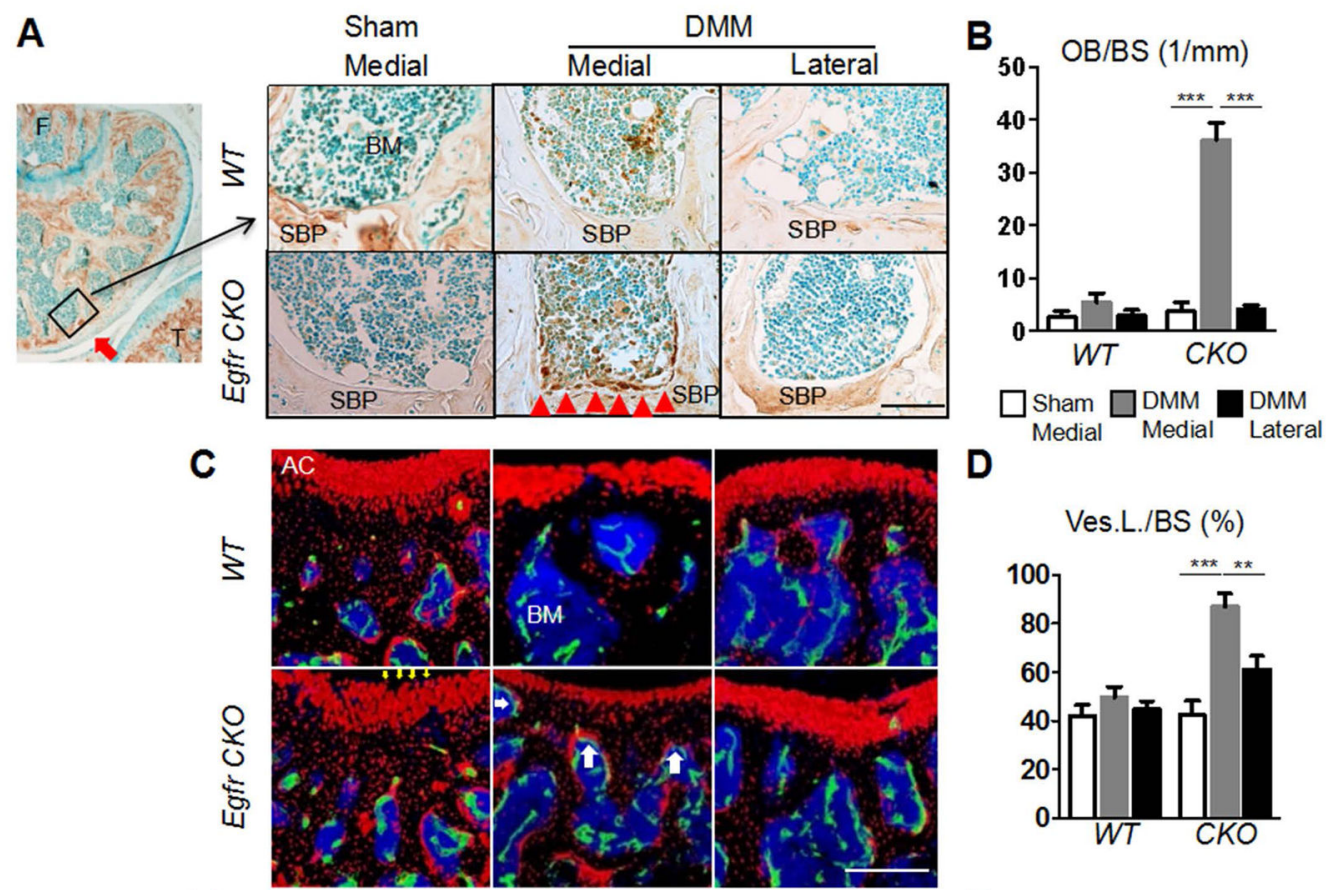

D Medial Medial Lateral

$\mathbf{E}$

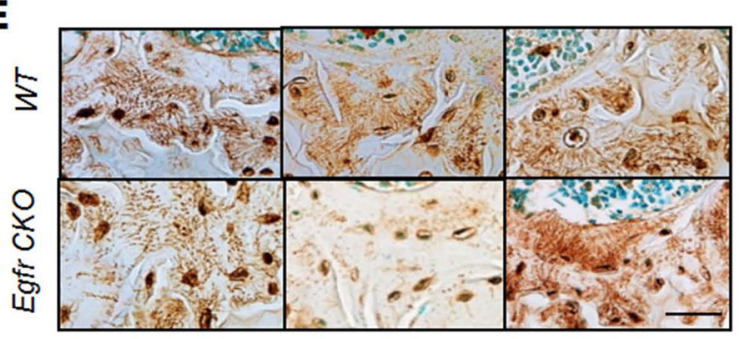

Ves.L./BS (\%)

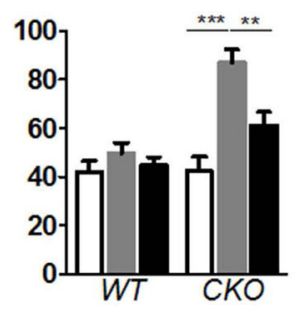

$\mathbf{F}_{150}$ Sclerostin intensity

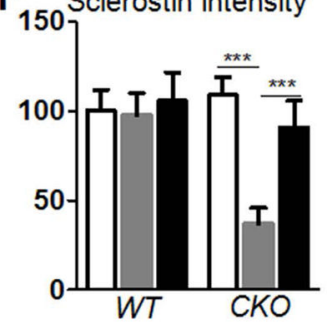

Figure 3.

DMM knees of Egfr CKO mice have escalated bone formation activity beneath the thickened SBP and a reduction of Sclerostin in the SBP.

(A). IHC of osteocalcin to identify osteoblasts beneath the SBP of WT and Egfr CKO mouse femurs at 2 months post-surgery. A low magnification image for the $W T$ sham medial section is presented on the left to show where the high magnification image on the right is cropped. Red triangles point to increased osteoblasts along the SBP. BM: bone marrow. Bar, $100 \mu \mathrm{m}$.

(B). The number of osteoblasts lining the SBP was quantified. $n=4$ mice/genotype. ***: $\mathrm{p}<0.001$.

(C). Representative confocal images of endomucin (green) staining to label bone marrow vasculature in $W T$ and Egfr $C K O$ joints. These mice also had Rosa-Tomato transgene so all bone marrow mesenchymal lineage cells, including bone lining osteoblasts, osteocytes, bone marrow mesenchymal progenitors and articular chondrocytes are Tomato ${ }^{+}$cells (red) ${ }^{(36)}$. White arrows point to vessels aligned along with the SBP surface. AC: articular cartilage. DAPI: blue. Scale bar: $150 \mu \mathrm{m}$.

(D). Quantification of the percentage of the SBP bone surface that is aligned with blood vessels. $\mathrm{n}=4$ /genotype. $* *$ : $\mathrm{p}<0.01, * * *$ : $\mathrm{p}<0.001$. 
(E). IHC of Sclerostin in the SBP. Bar, $30 \mu \mathrm{m}$.

(F). Quantification of Sclerostin staining intensity. $\mathrm{n}=4$ mice/genotype. ${ }^{* * *}: \mathrm{p}<0.001$. 

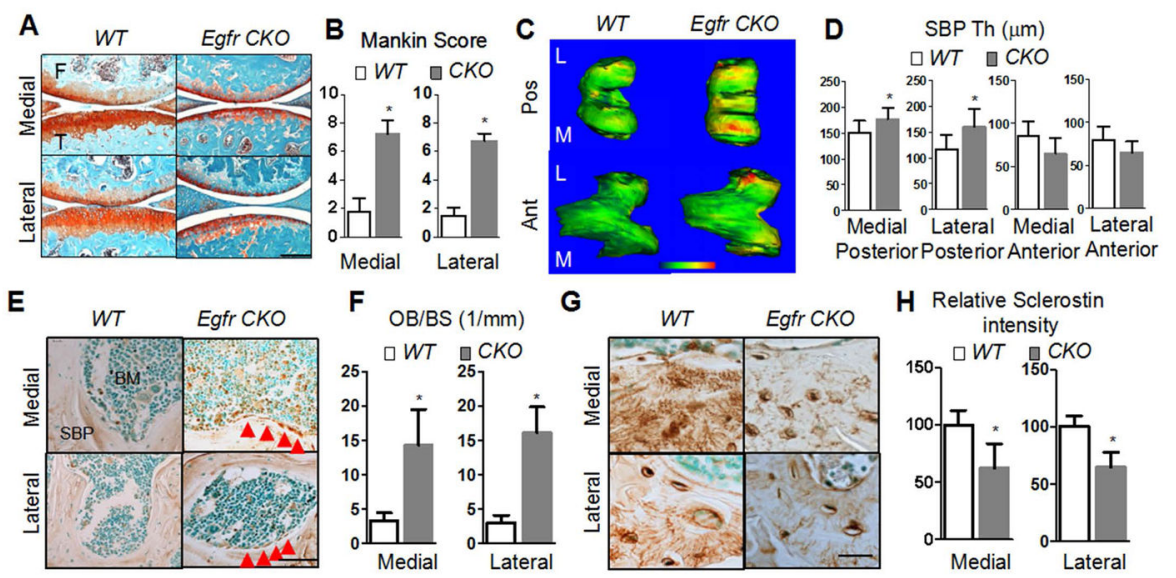

Figure 4.

SBP thickening in aged Egfr $C K O$ knees are also caused by increased bone formation along the SBP surface and suppressed Sclerostin level in the SBP.

(A). Safranin O staining of femoral epiphyseal region in 1-year-old WTand Egfr CKO mice. F: femur; T: tibia. Bar, $200 \mu \mathrm{m}$.

(B). The OA severity was measured by Mankin score. $\mathrm{n}=5$ mice/genotype. $*$ : $\mathrm{p}<0.05$ vs $W T$. (C). Representative 3D color maps of SBP thickness in WT and Egfr CKO femurs. Color ranges from 0 (blue) to $300 \mu \mathrm{m}$ (red).

(D). Quantification of SBP thickness (Th.) at four distinct femoral sites. $\mathrm{n}=9-11$ mice/ genotype. *: $\mathrm{p}<0.05$ vs $W T$.

(E). IHC of osteocalcin to identify osteoblasts beneath the SBP in $W T$ and Egfr $C K O$ mice. Red triangles point to increased osteoblasts along the SBP surface. BM: bone marrow. Bar, $100 \mu \mathrm{m}$.

(F). The number of osteoblasts lining the SBP surface was quantified. L: lateral; M: medial. n=4/genotype. *: p<0.05 vs $W T$.

(G). IHC of Sclerostin in the SBP of aged WT and Egfr CKO mice. Bar, $30 \mu \mathrm{m}$.

(H). Quantification of Sclerostin staining intensity demonstrates that Sclerostin amount is reduced at both medial and lateral sites of Egfr $C K O$ knee. n=4/genotype. *: p<0.05 vs WT. 

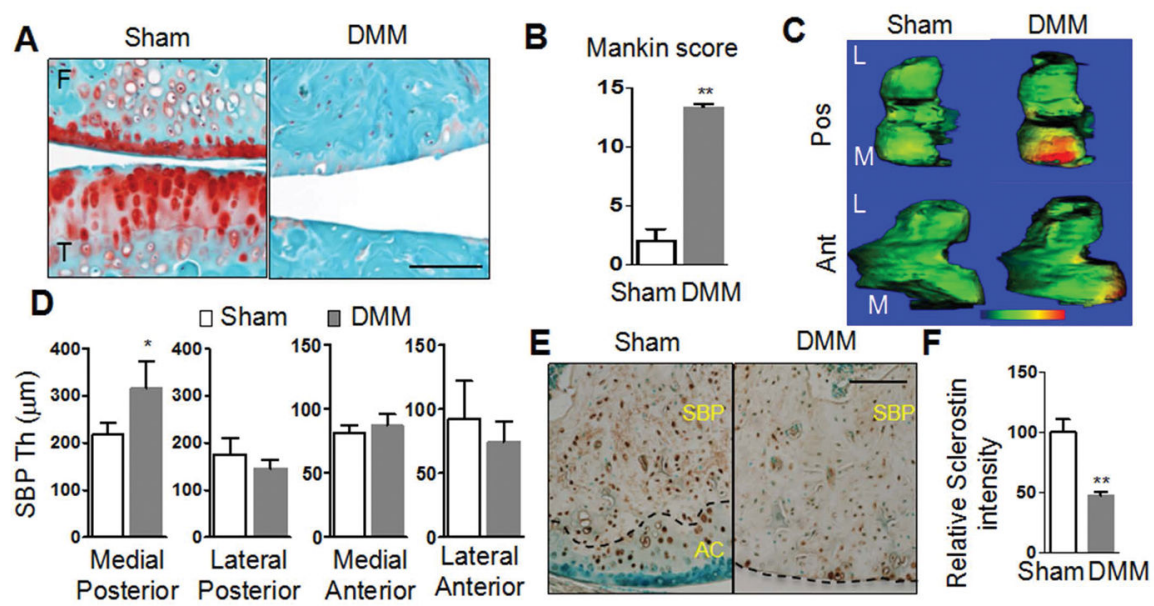

Figure 5.

Severe cartilage degeneration in $W T$ joints after long term post DMM is accompanied by substantial SBP thickening and suppression of Sclerostin within the SBP.

(A). Safranin O staining of WT mouse joints at 10 months post sham or DMM surgery. F: femur; T: tibia. Bar, $100 \mu \mathrm{m}$.

(B). The OA severity was measured by Mankin score. $\mathrm{n}=3$ mice/group. **: $\mathrm{p}<0.01$ vs sham.

(C). Representative 3D color maps of SBP thickness in Sham and DMM femurs. Color ranges from 0 (blue) to $420 \mu \mathrm{m}$ (red). Pos: posterior; Ant: anterior.

(D). Quantification of SBP thickness at 4 distinct femoral sites. $n=3$ mice/group. *: $\mathrm{p}<0.05$ vs sham.

(E). Sclerostin IHC staining at the medial posterior site of the SBP. AC: articular cartilage. Dashed line indicates the chondral-osseous junction. Bar, $100 \mu \mathrm{m}$.

(F). Quantification of Sclerostin staining intensity. $\mathrm{n}=3$ mice/group. **: $\mathrm{p}<0.01$ vs sham. 

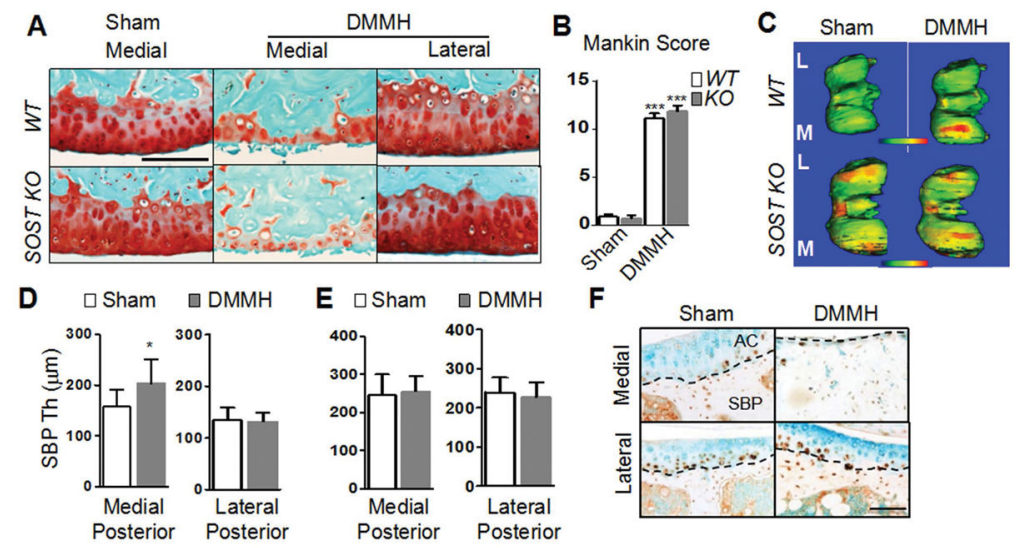

Figure 6.

DMMH surgery results in a thickened SBP at the late stage of OA in $W T$, but not in $S O S T$ $\mathrm{KO}$ mice.

(A). Safranin O staining of $W T$ and SOST KO mouse joints at 14 weeks post sham or DMMH surgery. Bar, $100 \mu \mathrm{m}$.

(B). The OA severity at DMMH medial posterior site was measured by Mankin score. $\mathrm{n}=6-7$ mice/group. ***: $\mathrm{p}<0.001$ vs sham.

(C). Representative 3D color maps of SBP thickness in WT and SOST KO femurs after sham and DMMH surgery. Color ranges from 0 (blue) to $282 \mu \mathrm{m}$ (red) for $W T$ and from 0 (blue) to $354 \mu \mathrm{m}$ (red) for SOST KO mice.

(D). Quantification of SBP thickness at medial posterior and lateral posterior sites of $W T$ mice. $\mathrm{n}=8$ mice/group. ${ }^{*}: \mathrm{p}<0.05$ vs sham.

(E). Quantification of SBP thickness at medial posterior and lateral posterior sites of $S O S T$ $K O$ mice. $\mathrm{n}=7$ mice/group.

(F). Sclerostin staining of $W T$ knees revealed strong staining in osteocytes within the SBP at sham medial, sham lateral and DMMH lateral sites, but not in those cells at DMMH medial site. Dashed line indicates the chondral-osseous junction. AC: articular cartilage. Bar, 100 $\mu \mathrm{m}$. 